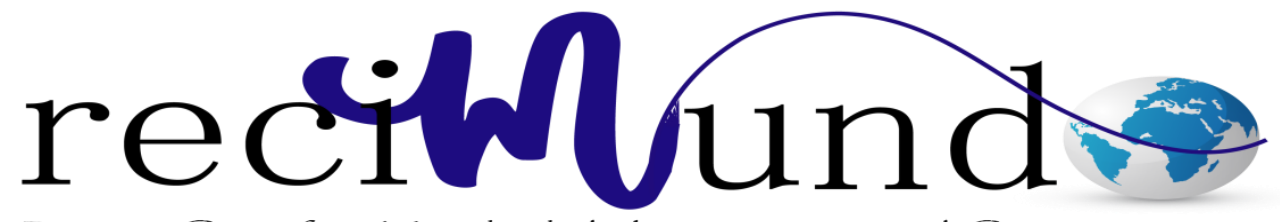

Revista Cientifica Mundo de la Investigación y el Conocimiento

Silvia Paulina Puente Tituaña ${ }^{\text {a; }}$ Alexandra del Rocio Canizares Stay ${ }^{\text {b; }}$

Betzabeth Johanna Solís Marín ${ }^{\mathrm{c}}$

Responsabilidad social en empresas industriales de Guayaquil

Revista Científica Mundo de la Investigación y el Conocimiento. Vol. 2 núm.2, mayo, ISSN: 2588-073X, 2018, pp. 650-667

DOI: 10.26820/recimundo/2.(2).2018.650-667

Editorial Saberes del Conocimiento

Recibido: 05/12/2017_ Aceptado: 18/03/2018
a. Universidad de Guayaquil; silvia.puentet@ug.edu.ec
b. Universidad de Guayaquil; alexandra.canizarezst@ug.edu.ec
c. Universidad de Guayaquil; betzabeth.solism@ug.edu.ec 


\section{Responsabilidad social en empresas industriales de Guayaquil}

Vol. 2, núm. 2., (2018)

Silvia Paulina Puente Tituaña; Alexandra del Rocio Canizares Stay; Betzabeth Johanna Solís

Marín

\section{RESUMEN}

En Guayaquil las empresas industriales tienen un desarrollo importante, social y económicamente muy activa; pues representa la base económica de la ciudad. En las últimas décadas las empresas a nivel mundial han tenido que aplicar sus operaciones a la situación actual de mayor compromiso con el medio ambiente y la sociedad en su conjunto, las empresas tiene el compromiso de reorientar sus beneficios económicos hacia nuevos objetivos de la responsabilidad social empresarial (RSE). Esta investigación se constituye en un indicador el cual permite conocer el alcance que tiene la responsabilidad social en empresas industriales de Guayaquil. Cada una de las citas cuenta con el sustento bibliográfico que ayuda a mejorar la comprensión del lector, es decir, toda esta visión desde una revisión bibliográfica.

Palabras Claves: Base económica de Guayaquil, medio ambiente, responsabilidad social, empresas industriales. 


\title{
Responsabilidad social en empresas industriales de Guayaquil
}

Vol. 2, núm. 2., (2018)

Silvia Paulina Puente Tituaña; Alexandra del Rocio Canizares Stay; Betzabeth Johanna Solís Marín

\begin{abstract}
In Guayaquil, industrial companies have an important social and economic development that is very active; because it represents the economic base of the city. In recent decades companies worldwide have had to apply their operations to the current situation of greater commitment to the environment and society as a whole, companies are committed to reorienting their economic benefits towards new objectives of social responsibility business (SRB). This research constitutes an indicator which allows knowing the scope of social responsibility in industrial companies in Guayaquil. Each of the citations has a bibliographical support that helps to improve the reader's understanding, that is, all this vision from a bibliographic review.
\end{abstract}

Key words: Economic base of Guayaquil, environment, social responsibility, industrial companies. 


\section{Responsabilidad social en empresas industriales de Guayaquil}

Vol. 2, núm. 2., (2018)

Silvia Paulina Puente Tituaña; Alexandra del Rocio Canizares Stay; Betzabeth Johanna Solís

Marín

\section{Introducción.}

En las últimas décadas las empresas a nivel mundial han tenido que adaptar sus operaciones a la situación actual de mayor vínculo con el medio ambiente y la sociedad en su conjunto, las empresas tienen el compromiso de reorientar sus beneficios económicos hacia nuevos objetivos de la responsabilidad social empresarial (RSE). Misma que de cara al siglo XXI es un campo de estudio muy amplio de gran trascendencia, para las empresas en el proceso de economías sostenibles.

No existe una fecha exacta sobre los inicios de la responsabilidad social, (EXPOK, 2016) indica que existen varios indicios que llevan a los estudiosos de este campo a deducir que, en el siglo XIX aparecen los primeros documentos que constatan las primeras prácticas de las empresas en pro de sus trabajadores.

Con el afán de cada vez crear mayor conciencia social y medioambiental se desarrollan un sin número de conferencias a nivel mundial, se fundan instituciones en pro de los derechos del medio ambiente, el cuidado de la tierra y todos los recursos naturales y animales, luego se crean organizaciones que siguen vigentes hasta la actualidad para dar soporte a todo lo que hoy en día se conoce como Responsabilidad Social Empresarial. Entre los acontecimientos más importantes que se han dado están: en 1971 la Conferencia de Estocolmo, en 1987 el Informe Brundtland, en 1997 el Protocolo de Kyoto, la fundación de grupos civiles como WWF, Amnistía Internacional, Greenpeace, a nivel empresarial iniciativas como AA1000, SA8000, el Pacto Mundial y organizaciones como la Global ReportingInitiative o el Instituto Ethos (EXPOK, 2016). 


\section{Responsabilidad social en empresas industriales de Guayaquil}

Vol. 2, núm. 2., (2018)

Silvia Paulina Puente Tituaña; Alexandra del Rocio Canizares Stay; Betzabeth Johanna Solís Marín

En 1999 el secretario general de la ONU durante el Foro Económico y Mundial de Davos pidió que se adoptasen valores con rostro humano al mercado mundial. Lo cierto es que desde los años noventa este concepto ha ido cobrando fuerza y evolucionado constantemente, tras el advenimiento de la globalización, el aceleramiento de la actividad económica, la conciencia ecológica y el desarrollo de nuevas tecnologías. Correa, (2004)

\section{Desarrollo}

\section{La empresa industrial y su impacto social}

Según Cannon (1994), fue la revolución industrial la que centró la atención sobre la responsabilidad empresarial, dado el poder que tenían los nuevos procesos industriales para reestructurar las relaciones del antiguo régimen. La revolución industrial introdujo -y sigue introduciendo- cambios fundamentales en las relaciones entre individuos y grupos de la sociedad. La riqueza de los propietarios y la magnitud de sus empresas levantaron sospechas respecto de su poder, sus motivaciones y sus métodos, e incluso llevaron a cuestionar los valores que configuraban su conducta y la ética con que operaban.

Rachman, et al (1991), indican que a medida que crecía el impacto de la industrialización sobre el ambiente natural o modificado, se convirtió en nuevo motivo de preocupación y debate. Las condiciones en torno a las fábricas y en las ciudades despertaron la ira en muchos y una onda preocupación en otros. Ya en la década de los setenta, el activismo (social) explotó en cuatro frentes: la protección ambiental, la defensa nacional, el asociacionismo de consumidores para su auto protección y los derechos civiles. 


\section{Responsabilidad social en empresas industriales de Guayaquil}

Vol. 2, núm. 2., (2018)

Silvia Paulina Puente Tituaña; Alexandra del Rocio Canizares Stay; Betzabeth Johanna Solís

Marín

\section{Responsabilidad social}

La Norma ISO 26000 precisa que: "La Responsabilidad Social es la responsabilidad de una organización por los impactos de sus decisiones y actividades en la sociedad y en el medio ambiente, a través de una conducta transparente y ética que: contribuya con el desarrollo sostenible, incluyendo la salud y el bienestar de la sociedad; Tome en cuenta las expectativas de las partes interesadas (stakeholders); Cumpla con las leyes y sea compatible con las normas internacionales de conducta; Sea integrada en la totalidad de la organización y puesta en práctica en todas sus relaciones" (ISO, 2010).

Pachi y Arbex, (2006), indican que el Instituto ETHOS de Empresas de Responsabilidad Social - Brasil, define como: “ RSE es una forma de gestión que se define por la relación ética de la empresa con todos los públicos con los cuales ella se relaciona, y por el establecimiento de metas empresariales compatibles con el desarrollo sustentable de la sociedad, preservando recursos ambientales y culturales para las generaciones futuras, respetando la diversidad y promoviendo la reducción de las desigualdades sociales”.

Un estudio del BID señala que: “Conviene tener en cuenta que la RSE es una temática que no nace desde la base empresarial propia latinoamericana, si no que se trata más bien de un tema importado por parte de las filiales de las grandes multinacionales extranjeras al que, progresivamente, las empresas nacionales (especialmente las grandes) y los gobiernos nacionales están prestando una atención creciente... Desgraciadamente, el conocimiento existente sobre la temática de RSE desde la perspectiva pyme puede calificarse de escasa y muy parcial, donde la 


\section{Responsabilidad social en empresas industriales de Guayaquil}

Vol. 2, núm. 2., (2018)

Silvia Paulina Puente Tituaña; Alexandra del Rocio Canizares Stay; Betzabeth Johanna Solís Marín

disponibilidad de informes comparativos entre países y basados en una metodología común es prácticamente inexistente”. Vives, Corral, (2005),

IRSE es la primera institución en el Ecuador en abordar, de manera técnica y metodológica, la responsabilidad social empresarial. Se cimenta en una filosofía de valores trascendentes, con aplicaciones prácticas, que guían a las organizaciones en la implementación de la RSE con base en metodologías, sistemas e iniciativas medibles, cuantificables, universalmente reconocidas y respetadas. Apuntalamos con nuestra gestión el desarrollo sostenible.

Podemos concluir en que es muy importante que las empresas tengan un Plan De Responsabilidad Social Corporativa, ya que estos les traerá muchos beneficios y les ayudará a optimizar la inversión socialmente responsable y ayudará al crecimiento del nivel de competitividad de la empresa. Un buen ejemplo de esto es la empresa PRONACA en donde se toma muy en serio su Responsabilidad Social la cual les ha llevado a ser reconocidas como una de las empresas cuya identificación del capital humano esta alineada con la estrategia de la empresa. Pronaca, (2013).

En la actualidad el Revised OECD Guidelines for Multinational Enterprises es el único código de conducta multilateralmente adoptado, aunque las directrices no definen expresamente el concepto de responsabilidad social corporativa, entre sus objetivos se menciona el de "Potenciar el aporte de las empresas multinacionales al desarrollo sostenible para garantizar una coherencia entre los objetivos sociales, económicos y ambientales” Acción Empresarial, (2006), citado por Castillo, Espinoza, (2017). 


\section{Responsabilidad social en empresas industriales de Guayaquil}

Vol. 2, núm. 2., (2018)

Silvia Paulina Puente Tituaña; Alexandra del Rocio Canizares Stay; Betzabeth Johanna Solís

Marín

Según World Economic Forum (WEF) este documento enfatiza 4 acciones necesarias para impulsar la Ciudadanía Corporativa Global: "Primero, proporcionar liderazgo. Segundo, definir qué quiere decir para su empresa. Tercero, hacerlo realidad. Cuarto, ser trasparente sobre ello”. Acción Empresarial, (2006), citado por Castillo, Espinoza, (2017).

El marco de acción propuesto por WEF deja en claro que existe una dificultad para precisar un concepto con contenido único para todos, por ello recomienda que cada empresa, junto a sus grupos de interés o stakeholders, reflexione sobre lo que la RSE significa para ellos. Acción Empresarial, (2006), citado por Castillo, Espinoza, (2017).

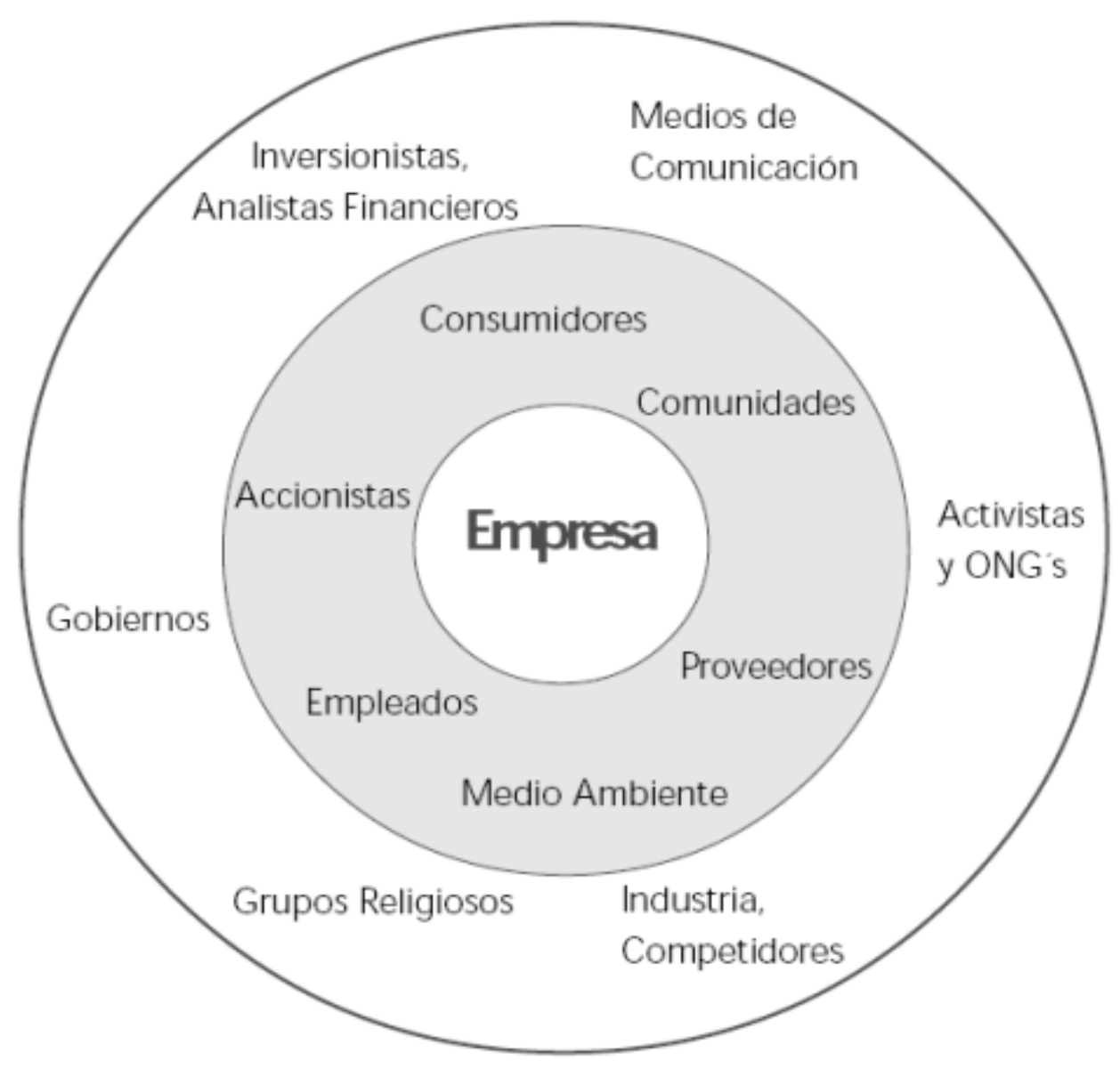

Revista Científica Mundo de la Investigación y el Conocimiento. 2 (2). pp. 650-667 


\section{Responsabilidad social en empresas industriales de Guayaquil}

Vol. 2, núm. 2., (2018)

Silvia Paulina Puente Tituaña; Alexandra del Rocio Canizares Stay; Betzabeth Johanna Solís Marín

\section{Figura 1. Respuesta de las Empresas a las expectativas de la Sociedad. Fuente: Acción}

Empresarial, (2006), citado por Castillo, Espinoza, (2017).

Bajo el esquema de la figura 1, podemos reconocer que en la actualidad el concepto de RSE es reconocido en nuestro país, convirtiéndose en uno de los principales actores que implican compromiso y promoción del cambio en procesos que ofrecen e innovan importantes empresas que generan el cambio y mejora del medio ambiente de la sociedad en la que vivimos.

Contenidos éticos, políticos, sociales y ambientales relacionado con temáticas de responsabilidad social, los cuales son expresados como subdimensiones. En la tabla 1.

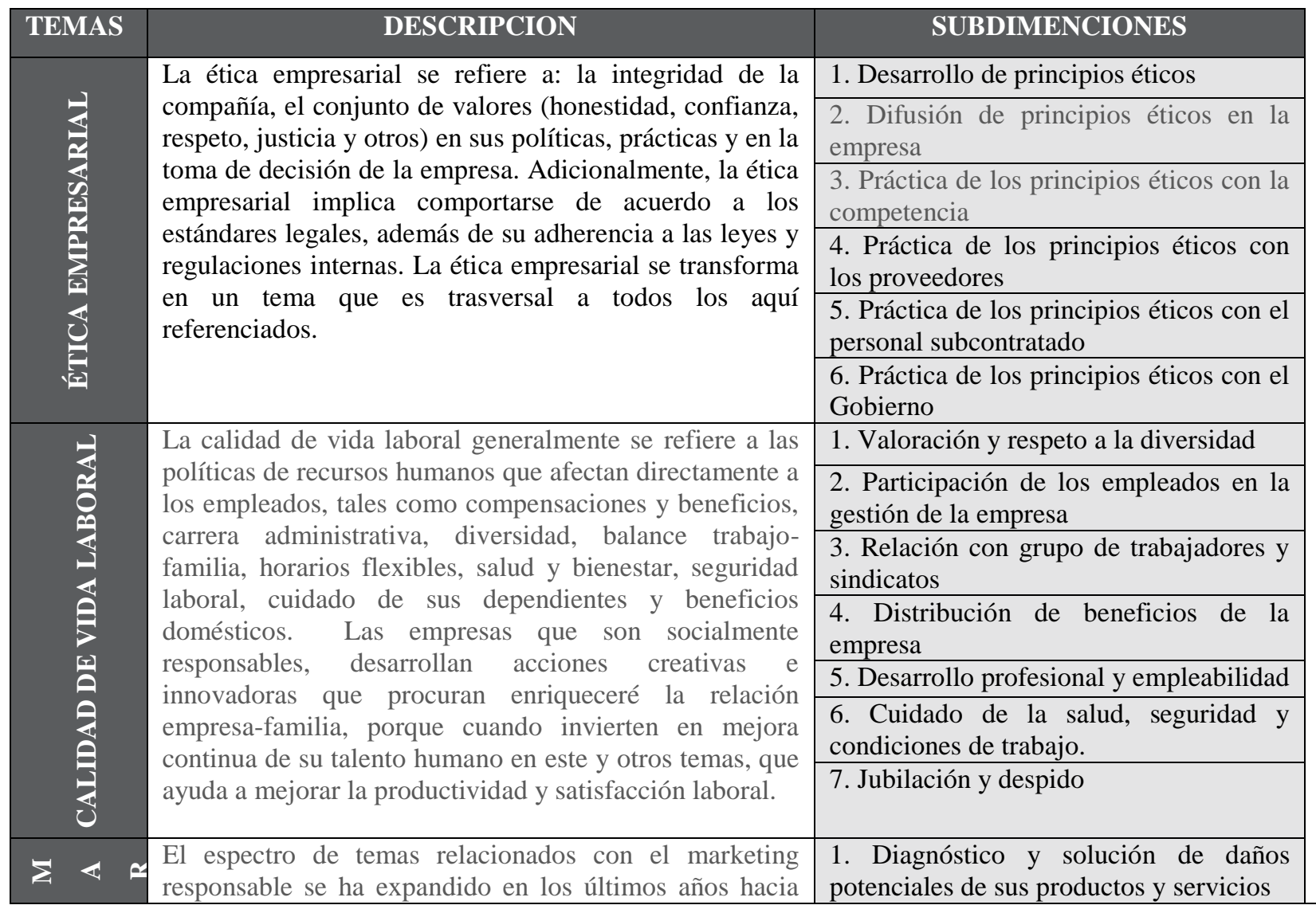




\section{Responsabilidad social en empresas industriales de Guayaquil}

Vol. 2, núm. 2., (2018)

Silvia Paulina Puente Tituaña; Alexandra del Rocio Canizares Stay; Betzabeth Johanna Solís

Marín

\begin{tabular}{|c|c|c|c|}
\hline \multirow{3}{*}{\multicolumn{3}{|c|}{$\begin{array}{l}\text { una amplia gama de tópicos que incluyen la } \\
\text { responsabilidad medio ambiental, la relación de la } \\
\text { empresa con sus competidores, y hacia temas algo más } \\
\text { filosóficos como el consumo de su propia naturaleza. Por } \\
\text { ello las empresas que son socialmente responsables, } \\
\text { buscan no afirmar su identidad sino también su imagen } \\
\text { en el mercado, motivando además el consumo } \\
\text { responsable. }\end{array}$}} & 2. Políticas d \\
\hline & & & $\begin{array}{l}\text { 3. Técnica de ventas éticas y respeto a la } \\
\text { privacidad del consumidor }\end{array}$ \\
\hline & & & $\begin{array}{l}\text { 4. Excelencia a la atención del } \\
\text { consumidor }\end{array}$ \\
\hline \multirow{7}{*}{\multicolumn{2}{|c|}{ 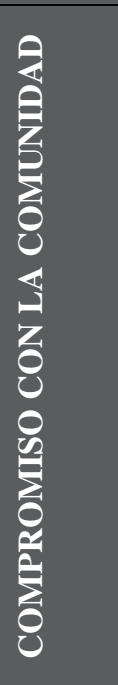 }} & \multirow{7}{*}{$\begin{array}{l}\text { Esta área se refiere al rango de acciones tomadas por la } \\
\text { empresa para mejorar la calidad de vida de las } \\
\text { comunidades en la que opera, apoyando iniciativas o } \\
\text { causas con enfoque social, estos programas se diseñan y } \\
\text { ejecutan estrategias y programadamente, no tan solo se } \\
\text { entrega un beneficio a los receptores, sino que además se } \\
\text { refuerza la reputación de las empresas y sus marcas, } \\
\text { productos y valores en la comunidades locales y en el } \\
\text { resto del mundo donde tienen intereses comerciales } \\
\text { significativos. Empresas ecuatorianas desarrollan } \\
\text { proyectos muy interesantes en la comunidad en lo } \\
\text { referente a educación, salud, asistencia técnica, } \\
\text { capacitación, con ello ayudan a controlar o en su defecto } \\
\text { a disminuir las tensiones que se pueden dar con la } \\
\text { comunidad, cuando ésta se ve afectada por la actividad } \\
\text { empresarial, tanto en su medio ambiente como en su } \\
\text { calidad de vida. }\end{array}$} & $\begin{array}{l}\text { 1. Gestión del impacto de las actividades } \\
\text { de la empresa con la comunidad }\end{array}$ \\
\hline & & & $\begin{array}{l}\text { 2. Relaciones con organizaciones } \\
\text { comunitarias presente en su entorno } \\
\text { (ONG's, colegios, salud, etc.) }\end{array}$ \\
\hline & & & $\begin{array}{l}\text { 3. Mecanismos de apoyo a proyectos } \\
\text { sociales. }\end{array}$ \\
\hline & & & $\begin{array}{l}\text { 4. Estrategias y criterios de acción en } \\
\text { inversiones sociales }\end{array}$ \\
\hline & & & $\begin{array}{l}\text { 5. Apoyo al trabajo voluntario del } \\
\text { personal }\end{array}$ \\
\hline & & & 6. Liderazgo e influencia social \\
\hline & & & $\begin{array}{l}\text { 7. Participación en proyectos sociales del } \\
\text { Gobierno }\end{array}$ \\
\hline & \multirow{4}{*}{$\frac{\sum_{0=}^{\infty}}{\sum_{\infty}^{\infty}}$} & \multirow{4}{*}{$\begin{array}{l}\text { Relacionado con los posibles impactos que la actividad } \\
\text { empresarial pueda causar tanto al medio ambiente natural } \\
\text { como al medio ambiente construido. La actividad } \\
\text { ambientalmente responsable, implica la aplicación de las } \\
\text { normas ambientales vigentes en el país así como las } \\
\text { internacionales como ISO 14.000. Proyecta para ello } \\
\text { también procesos de educación, prevención y } \\
\text { remediación. }\end{array}$} & 1. El impacto en el medio ambiente \\
\hline & & & 2. Responsabilidad ambiental \\
\hline & & & $\begin{array}{l}\text { 3. Cuidados e incorporación de inst } \\
\text { y tratamiento de desechos }\end{array}$ \\
\hline & & & 4. Educación ambiental. \\
\hline
\end{tabular}

\section{TABLA 1. Áreas temáticas y subdimensiones de la RSE, Fuente: Barba-Bayas (2015), citado por Castillo, Espinoza, (2017).}

Duque, S. y Peña A., (2013), determina que la investigación realizada expone a las empresas privadas en su mayoría como elementos irresponsables dentro del mercado nacional, observando en ellas la ausencia de prácticas y aspectos fundamentales para el bienestar de sus elementos humanos y para con el medio ambiente. Resume que la escasez de esta práctica se debe a los compromisos que trae en muchas ocasiones; requiere de inversiones económicas, organización de grupos de interés, auspicios y padrinazgos que al momento significan dinero, 


\section{Responsabilidad social en empresas industriales de Guayaquil}

Vol. 2, núm. 2., (2018)

Silvia Paulina Puente Tituaña; Alexandra del Rocio Canizares Stay; Betzabeth Johanna Solís Marín

pero a la larga se lo puede señalar como una provechosa inversión ya que el buen manejo de un sistema que obliga a la empresa privada a ser SOCIALMENTE RESPONSABLE ahorra costos en la gestión de residuos en contaminación con el medio ambiente y a su vez eleva el compromiso de sus empleados para con la empresa.

Padilla, et al (2017), Se aplicó el método de encuesta, un cuestionario cualitativocuantitativo con ítems medidos en escala Likert de cinco puntos para recoger información del empleo de prácticas socialmente responsables, a una muestra de 192 empresas localizadas en las ciudades de Quito y Guayaquil, Ecuador. Se determinó la existencia de correlación de las variables de RSE con los índices financieros. Además se observó que las empresas ecuatorianas muestran un nivel medio de implementación del concepto de RSE. Esta correlación no muestra dependencias estadísticamente significativas entre el índice de RSE y el desempeño financiero. De acuerdo a los resultados de este estudio, sí existe una relación significativa entre el pilar de responsabilidad social y el rendimiento sobre las ventas o activos (ROS), y entre los pilares de responsabilidad económica y ambiental con respecto al retorno sobre el patrimonio (ROE).

Según Castillo, Espinoza, (2017), Las empresas no informan los resultados positivos a sus grupos de interés que se ejecutan en su beneficio, para que no se aprovechen en pedir obligatoriamente apoyo económico para proyectos y actividades de su entorno. En este estudio se determinó que las industrias localizadas en el sector de Durán tienen una buena práctica de responsabilidad social, pero hay que crear conciencia que la RSE debe ser una acción voluntaria y que debe haber un compromiso permanente en lo que se refiere al desarrollo social, económico y de medio ambiente. En este estudio podemos percibir que los beneficios que tiene la práctica 


\section{Responsabilidad social en empresas industriales de Guayaquil}

Vol. 2, núm. 2., (2018)

Silvia Paulina Puente Tituaña; Alexandra del Rocio Canizares Stay; Betzabeth Johanna Solís

Marín

de RSE son a mediano plazo, debido al despertar de la sensibilización de los empresarios de las empresas de Durán para contribuir al desarrollo social de la comunidad.

Viteri M., J., (2010), manifiesta que, las empresas ecuatorianas se encuentran en una etapa de transición, en el rol que desempeñan dentro de la sociedad, al pasar de prácticas de ayuda social como beneficencia, a la RSE, que nace en los principios y valores organizacionales, enmarcados en una misión y visión, articulando estrategias para mejorar la situación del entorno y hacer que estas acciones perduren en el tiempo; así, en 1998 la Fundación Esquel Ecuador, pionera en la promoción de la RS, junto a otras organizaciones del País y el Synergos Institute de Estados Unidos, dan los primeros pasos en la creación del Consorcio Ecuatoriano para la Responsabilidad Social (Ceres), y en el año 2005 se constituye esta ONG, como miembro de la Red Continental de Forum Empresa.

Según un estudio desarrollado por Ceres, 8 de cada 10 ecuatorianos desconocen qué es la RSC. En este mismo estudio en Quito, se asocia a la RSC con acciones y compromisos de las empresas con el medio ambiente o el entorno comunitario y en Guayaquil con programas de ayuda a damnificados. La ONG británica Accountability, según el estudio "Estado de la Competitividad Responsable", ubica al Ecuador en la posición 79, en un ranking de 108 países. Viteri M., J., (2010).

Según Viteri M., J., (2010), En la cultura ecuatoriana se confunde el concepto de RSC como filantropía asociada al paternalismo y asistencialismo e identificado como una acción exclusiva de gente adinerada y, en el caso de las empresas, como una acción de marketing. En el país hay varios ejemplos de organizaciones que han adoptado a la RSE como una estrategia, que 


\section{Responsabilidad social en empresas industriales de Guayaquil}

Vol. 2, núm. 2., (2018)

Silvia Paulina Puente Tituaña; Alexandra del Rocio Canizares Stay; Betzabeth Johanna Solís Marín

les llevará a alcanzar no solo el éxito económico sino el más alto reconocimiento de calidad e identificación social, entre las que se pueden citar: REPSOL YPF Ecuador S.A., Ecuador Bottling Company (Coca Cola), Pronaca, Palmeras del Ecuador S.A., Holcim Ecuador, Nestlé Ecuador, Yambal Ecuador S.A., Supermercados La Favorita C.A.

La Constitución de la República del Ecuador es la base legal para el cumplimiento de la responsabilidad social en nuestro país, los títulos más notables son: Título II: Derechos, Título VI: Régimen de desarrollo y el Título VII: Régimen del buen vivir. Los dos últimos títulos mencionados vinculados constituyen reglas para institucionalizar los "Derechos".

El Título VI: Régimen de desarrollo; capítulo primero: principios generales, Art. 275.- El régimen de desarrollo es el conjunto organizado, sostenible y dinámico de los sistemas económicos, políticos, socio-culturales y ambientales, que garantizan la realización del buen vivir, del sumak kawsay.

El Estado planificará el desarrollo del país para garantizar el ejercicio de los derechos, la consecución de los objetivos del régimen de desarrollo y los principios consagrados en la Constitución. La planificación propiciará la equidad social y territorial, promoverá la concertación, y será participativa, descentralizada, desconcentrada y transparente.

El buen vivir requerirá que las personas, comunidades, pueblos y nacionalidades gocen efectivamente de sus derechos, y ejerzan responsabilidades en el marco de la interculturalidad, del respeto a sus diversidades, y de la convivencia armónica con la naturaleza. 


\section{Responsabilidad social en empresas industriales de Guayaquil}

Vol. 2, núm. 2., (2018)

Silvia Paulina Puente Tituaña; Alexandra del Rocio Canizares Stay; Betzabeth Johanna Solís

Marín

Capítulo segundo: planificación participativa para el desarrollo, capítulo tercero: soberanía alimentaria, capítulo cuarto: soberanía económica.

El Título VII: Régimen del buen vivir; capítulo primero: inclusión y equidad, Art. 340.EI sistema nacional de inclusión y equidad social es el conjunto articulado y coordinado de sistemas, instituciones, políticas, normas, programas y servicios que aseguran el ejercicio, garantía y exigibilidad de los derechos reconocidos en la Constitución y el cumplimiento de los objetivos del régimen de desarrollo.

El sistema se articulará al Plan Nacional de Desarrollo y al sistema nacional descentralizado de planificación participativa; se guiará por los principios de universalidad, igualdad, equidad, progresividad, interculturalidad, solidaridad y no discriminación; y funcionará bajo los criterios de calidad, eficiencia, eficacia, transparencia, responsabilidad y participación.

El sistema se compone de los ámbitos de la educación, salud, seguridad social, gestión de riesgos, cultura física y deporte, hábitat y vivienda, cultura, comunicación e información, disfrute del tiempo libre, ciencia y tecnología, población, seguridad humana y transporte.

Capítulo segundo: Biodiversidad y recursos naturales.

En cumplimento a la Ley Orgánica de Educación Superior, capítulo 2, Fines de la educación superior.

Art. 3.- Fines de la Educación Superior.- La educación superior de carácter humanista, cultural y científica constituye un derecho de las personas y un bien público social que, de 


\section{Responsabilidad social en empresas industriales de Guayaquil}

Vol. 2, núm. 2., (2018)

Silvia Paulina Puente Tituaña; Alexandra del Rocio Canizares Stay; Betzabeth Johanna Solís Marín

conformidad con la Constitución de la República, responderá al interés público y no estará al servicio de intereses individuales y corporativos.

Art. 4.- Derecho a la Educación Superior.- El derecho a la educación superior consiste en el ejercicio efectivo de la igualdad de oportunidades, en función de los méritos respectivos, a fin de acceder a una formación académica y profesional con producción de conocimiento pertinente y de excelencia.

Las ciudadanas y los ciudadanos en forma individual y colectiva, las comunidades, pueblos y nacionalidades tienen el derecho y la responsabilidad de participar en el proceso educativo superior, a través de los mecanismos establecidos en la Constitución y esta Ley.

Art. 8.- Serán Fines de la Educación Superior.- La educación superior tendrá los siguientes fines:

a) Aportar al desarrollo del pensamiento universal, al despliegue de la producción científica y a la promoción de las transferencias e innovaciones tecnológicas:

b) Fortalecer en las y los estudiantes un espíritu reflexivo orientado al logro de la autonomía personal, en un marco de libertad de pensamiento y de pluralismo ideológico;

c) Contribuir al conocimiento, preservación y enriquecimiento de los saberes ancestrales y de la cultura nacional; 


\section{Responsabilidad social en empresas industriales de Guayaquil}

Vol. 2, núm. 2., (2018)

Silvia Paulina Puente Tituaña; Alexandra del Rocio Canizares Stay; Betzabeth Johanna Solís

Marín

d) Formar académicos y profesionales responsables, con conciencia ética y solidaria, capaces de contribuir al desarrollo de las instituciones de la República, a la vigencia del orden democrático, y a estimular la participación social:

e) Aportar con el cumplimiento de los objetivos del régimen de desarrollo previsto en la Constitución y en el Plan Nacional de Desarrollo:

f) Fomentar y ejecutar programas de investigación de carácter científico, tecnológico y pedagógico que coadyuven al mejoramiento y protección del ambiente y promuevan el desarrollo sustentable nacional:

g) Constituir espacios para el fortalecimiento del Estado Constitucional, soberano, independiente, unitario, intercultural, plurinacional y laico: y.

h) Contribuir en el desarrollo local y nacional de manera permanente, a través del trabajo comunitario o extensión universitaria.

\section{Conclusiones.}

En la actualidad las empresas que desea alcanzar el éxito en sus procedimientos industriales y comerciales deben aplicar a sus procesos la responsabilidad social empresarial a su gestión, no solo en cumplimento con la ley, sino como un compromiso transparente y consciente del impacto que produce la actividad industrial al medio ambiente. Es importante reconocer que la tierra es la que nos proporciona todos los recursos que se utilizan en la ejecución de la producción, por lo tanto es vital su preservación para las futuras generaciones. 


\section{Responsabilidad social en empresas industriales de Guayaquil}

Vol. 2, núm. 2., (2018)

Silvia Paulina Puente Tituaña; Alexandra del Rocio Canizares Stay; Betzabeth Johanna Solís Marín

Se puede determinar que existen empresas privadas que actúan irresponsablemente en el mercado nacional por cuanto se observa en ellas el abandono criterios fundamentales y prácticas para el bienestar de sus elementos humanos y para con el medio ambiente.

Conviene indicar que la cultura ecuatoriana confunde el concepto responsabilidad social empresarial como filantropía asociada al paternalismo y asistencialismo e identificando como una acción exclusiva de gente adinerada y, en el caso de las empresa, como una acción de marketing. Sin embargo existen empresas que han comprometido a ejecutar sus operaciones con RSE como una estrategia, que no solo les llevara a alcanzar el éxito económico sino también el reconocimiento de calidad e identificación social ante la comunidad.

Se puede concluir indicando que la ausencia de conocimiento sobre la responsabilidad social empresarial, es el principal efecto de la pobre aplicación en las empresas privadas del sector industrial de Guayaquil.

\section{Referencias Bibliográficas.}

Cannon, T., (1994), “La responsabilidad de la empresa”, Ediciones Folio, Barcelona.

Castillo, G., Espinoza, W., (2017), "Responsabilidad Social Empresarial: Diagnostico en la zona industrial de Duran”, Revista Publicando, 4 No 10, 24-43. ISSN 1390-9304.

Correa, M.E., (2004), "Responsabilidad social empresarial: una nueva forma de hacer negocios, revista futuros", No. 6, Vol. II.

Duque V., Sandra y Peña G., Álvaro, (2013), “Responsabilidad social en empresas privadas del sector industrial agropecuario de la ciudad de Guayaquil: Diagnostico y situación actual”, Tesis, Universidad Politécnica Salesiana, Ecuador.

EXPOK (2016), “Comunicación de sustentabilidad y RSE”, Recuperado de http://www.expoknews.com 


\section{Responsabilidad social en empresas industriales de Guayaquil}

Vol. 2, núm. 2., (2018)

Silvia Paulina Puente Tituaña; Alexandra del Rocio Canizares Stay; Betzabeth Johanna Solís

Marín

IRSE, (2005), http://irse-ec.org/quienessomos.html

ISO, S. G. (2010). Norma Internacional ISO 26000. Ginebra: Traslation Management Group.

Pachi, F., Arbex, N. (2006). “Conceptos Básicos e Indicadores de Responsabilidad Social Empresarial”. Obtenido de Instituto Ethos, la Fundación EMPRENDER y REDES: http://www1.ethos.org.br/EthosWeb/arquivo/0-A-77fREDES_1.pdf

Padilla Carmen, Arévalo Danny, Bustamante Miguel, Vidal Cristian, (2017), "Responsabilidad Social Empresarial y Desempeño Financiero en la Industria del Plástico en Ecuador”, https://scielo.conicyt.cl/scielo.php?script=sci_arttext\&pid=S0718

Pronaca, (2013), “Pronaca Responsabilidad Social”. Recuperado: www.pronaca.com

Rachman, D. J.; Mescon, M.H.; Bovée, C. L. y Thill, J.V., (1991), “Introducción a los negocios", Sexta Ed., Mc-Graw Hill.

Registro Oficial N 449, (2008), Constitución de la República del Ecuador, 20 de octubre.

Registro Oficial Suplemento No 298, (2010), Ley Orgánica de Educación Superior, Título I, Capítulo II, Fines de la Educación Superior, (Arts. 3, 4, 8), 12 de octubre.

Viteri M., J., (2010), “Responsabilidad social”, Universidad Tecnológica Equinoccial, ENFOQUTE. 1: 90-100, ISSN: 1390-6542.

Vives, A., Corral, A., (2005), "Responsabilidad Social en las pymes de Latinoamérica", BID, Washington DC. 